\title{
Human regulatory $T$ cells undergo self-inflicted damage via granzyme pathways upon activation
}

Esilida Sula Karreci, ${ }^{1}$ Siawosh K. Eskandari, ${ }^{1}$ Farokh Dotiwala, ${ }^{2}$ Sujit K. Routray, ${ }^{1}$ Ahmed T. Kurdi, ${ }^{3}$ Jean Pierre Assaker, ${ }^{1}$ Pavlo Luckyanchykov, ${ }^{1}$ Albana B. Mihali, ${ }^{1}$ Omar Maarouf, ${ }^{1}$ Thiago J. Borges, ${ }^{1}$ Abdullah Alkhudhayri, ${ }^{1}$ Kruti R. Patel, ${ }^{4}$ Amr Radwan, ${ }^{1}$ Irene Ghobrial, ${ }^{3}$ Martina McGrath, ${ }^{1}$ Anil Chandraker, ${ }^{1}$ Leonardo V. Riella, ${ }^{1}$ Wassim Elyaman, ${ }^{4}$ Reza Abdi, ${ }^{1}$ Judy Lieberman, ${ }^{2}$ and Jamil Azzi ${ }^{1}$

'Transplantation Research Center, Renal Division, Brigham and Women's Hospital and Children's Hospital, ${ }^{2}$ Program in Cellular and Molecular Medicine, Boston Children's Hospital, ${ }^{3}$ Department of Medical Oncology, Dana-Farber Cancer Institute, and ${ }^{4}$ Ann Romney Center for Neurologic Diseases, Brigham and Women's Hospital, Harvard Medical School, Massachusetts, USA.

Tregs hold great promise as a cellular therapy for multiple immunologically mediated diseases, given their ability to control immune responses. The success of such strategies depends on the expansion of healthy, suppressive Tregs ex vivo and in vivo following the transfer. In clinical studies, levels of transferred Tregs decline sharply in the blood within a few days of the transfer. Tregs have a high rate of apoptosis. Here, we describe a new mechanism of Treg self-inflicted damage. We show that granzymes $A$ and -B (GrA and GrB), which are highly upregulated in human Tregs upon stimulation, leak out of cytotoxic granules to induce cleavage of cytoplasmic and nuclear substrates, precipitating apoptosis in target cells. GrA and GrB substrates were protected from cleavage by inhibiting granzyme activity in vitro. Additionally, we show - by using cytometry by time of flight (CYTOF) - an increase in GrB-expressing Tregs in the peripheral blood and renal allografts of transplant recipients undergoing rejection. These GrB-expressing Tregs showed an activated phenotype but were significantly more apoptotic than non-GrB expressing Tregs. This potentially novel finding improves our understanding of Treg survival and suggests that manipulating Gr expression or activity might be useful for designing more effective Treg therapies.

Authorship note: ESK, SKE, FD, and SKR contributed equally to this work

Conflict of interest: The authors have declared that no conflict of interest exists.

Submitted: November 3, 2016 Accepted: September 25, 2017 Published: November 2, 2017

\section{Reference information:}

JCI Insight. 2017;2(21):e91599. https://doi.org/10.1172/jici. insight.91599.

\section{Introduction}

Tregs are vital to the maintenance of naturally occurring and induced peripheral immune tolerance. Given their capacity to suppress immune reactivity against self and alloantigens, manipulation of this $\mathrm{T}$ helper cell subset is a promising strategy for treating autoimmune disease, graft-versus-host disease (GvHD) and to promote transplantation tolerance (1-10). Tregs suppress effector cells via multiple mechanisms, including secretion of soluble factors (IL-10, TGF- $\beta$ ); upregulation of checkpoint inhibitory molecules, such as CTLA-4; and through direct granzyme-mediated (Gr-mediated) cytolysis of effector cells $(11,12)$. In mice, Treg-secreted $\mathrm{GrB}$ has been shown to improve allograft survival significantly $(13,14)$.

Infusions of ex vivo-expanded Tregs are being investigated in clinical trials as a cellular therapy to prevent or treat transplant rejection, GvHD, and autoimmune diseases, including type 1 diabetes and systemic lupus erythematosus $(5-7,9)$. Despite their promise, a key observation in these studies is the short lifespan of adoptively transferred Tregs $(5,6,9,15)$. Brunstein et al. showed that infused Treg numbers peaked 2 days after infusion and were detectable for only 14 days (5). In nonhuman primates, Treg half-life was less than 14 days, despite the use of a Treg-promoting immunosuppressive regimen that included rapamycin (15). The cause of the rapid loss of ex vivo-expanded Tregs has not yet been fully explained. However, significant improvements in Treg expansion and adoptive transfer have been achieved recently where most of the Treg expansion protocols used rapamycin (16-19). Bluestone and colleagues recently showed, using deuterium labeling, persistence of Tregs for months after adoptive transfer by measuring it over time. However, the DNA enrichment was less than $20 \%$ of the peak level a few days after the adoptive transfer (20), which could either be due to Treg death or to their migration from the blood. 
Merged
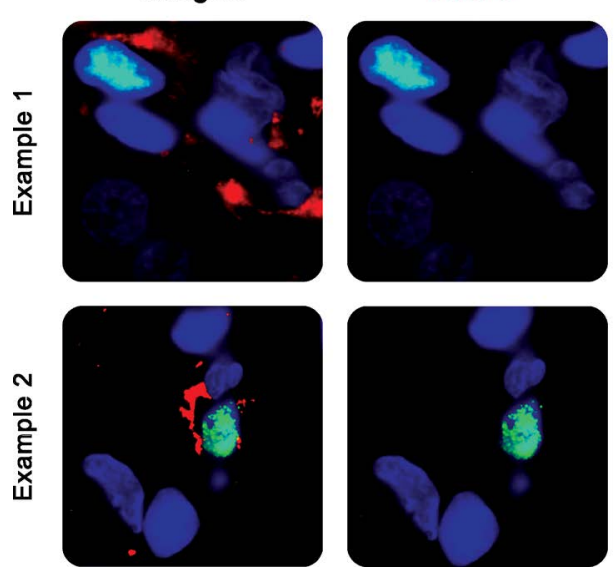

Figure 1. GrB expression among Tregs in the renal allograft of a transplant recipient undergoing rejection. Two representative confocal microscopy images $(63 x)$ of a kidney allograft biopsy of a patient undergoing clinical rejection. We observed that Tregs characterized by nuclear FoxP3 staining (FITC color, green) coexpressed with cytoplasmic GrB staining (PE color, red). FoxP3, forkhead box P3.

Granzyme B
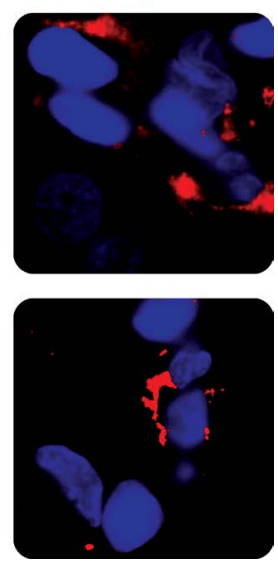

Grs induce programmed cell death in cells targeted for elimination by immune killer lymphocytes, including NK cells and $\mathrm{CD}^{+} \mathrm{T}$ cells $(11,21,22)$. Some CD4 T cells, especially Th1 cells, innate-like NKT cells, and $\gamma \delta \mathrm{T}$ cells also have the capacity to kill $(22,23)$. Killer cells are protected from self-destruction in part by concurrent expression of serine protease inhibitors (serpins) (24). Serpins tightly bind serine proteases, including the Grs, to form complexes that irreversibly inactivate these proteases (25). Serine protease inhibitor 6 (Spi6) protects murine cytotoxic $\mathrm{T}$ cells and DCs from GrB-induced cytotoxicity $(26,27)$. The human equivalent, proteinase inhibitor-9 (PI9) is upregulated with $\mathrm{GrB}$ in human leukocytes, but the effect of PI9 on human Treg function and survival has not yet been established. Specific intracellular serpins that inactivate the other Grs in Gr-expressing cells are unknown (28).

When murine Tregs are activated, they express high levels of GrB $(11,13)$. Human Tregs express both GrB and GrA, which have been shown to contribute to control of T effector cells (21). Although Grs and killing of autoreactive or alloreactive T cells may be important in immune suppression, Gr expression could lead to self-inflicted Treg death. In this study, we investigated whether Grs might play a role in the short lifespan of activated Tregs. We found that GrA and GrB are highly upregulated in human Tregs upon stimulation. Grs leak out of intracellular granules and induce cleavage of cytoplasmic, mitochondrial, and nuclear targets, resulting in cell apoptosis. We also show using cytometry by time of flight (CYTOF) that, among kidney transplant recipients undergoing rejection, there is an increase in GrB-expressing Tregs in the periphery and within the renal allograft. GrB-expressing Tregs showed an activated phenotype but were significantly more apoptotic than non-GrB-producing Tregs.

\section{Results}

Tregs in patients undergoing kidney allograft rejection express GrB. Renal allograft biopsies were obtained from transplant recipients undergoing renal allograft biopsy for increased serum creatinine. In allograft biopsies of patients undergoing cellular rejection, FoxP3 ${ }^{+}$Tregs expressed GrB (Figure 1). This finding suggests that GrB expression in Tregs could be associated with graft rejection.

$\mathrm{GrB}^{+}$Tregs express coinhibitory and chemokine receptors that mediate trafficking to sites of inflammation. To understand the differences between $\mathrm{GrB}^{+}$and $\mathrm{GrB}^{-}$Tregs $\left(\mathrm{CD} 4^{+} \mathrm{CD} 25^{\text {high }} \mathrm{CD} 127^{\text {low }} \mathrm{FoxP}^{+}\right)$in allograft rejection, PBMCs from kidney transplant recipients undergoing rejection were analyzed using mass cytometric CyTOF (Figure 2). $\mathrm{GrB}^{+}$compared with $\mathrm{GrB}^{-}$Tregs showed significantly greater expression of CTLA4 and PD-L1, with a trend toward significance in TIM3. GrB ${ }^{+}$Tregs also showed a significant increase in the expression of the chemokine receptors CXCR3 and CCR6, which regulate Treg migration into areas of inflammation mediated by Th1 and Th17, respectively (29-32) (Figure 2, D and E). The Tregs also significantly overexpressed the antiapoptotic molecule, BCL-2, which inhibits caspase-mediated death activity by GrB (Figure 2, D and E) (33-35). Spanning-Tree Progression Analysis of Density-Normalized Events (SPADE) was used to portray the differences in expression of the differentially expressed proteins within the 2 subpopulations of $\mathrm{GrB}^{+}$and $\mathrm{GrB}^{-}$Tregs (Figure 2E). Each SPADE tree represents data from 1 patient. Each node of the SPADE tree represents a cell population, while the size of a node corresponds to the number of cells per population, and the node color indicates the signal intensity of the respective markers. A significant increase in the expression of some coinhibitory molecules and chemokine receptors was observed on $\mathrm{GrB}^{+}$compared with $\mathrm{GrB}^{-}$Tregs. However, the level of expression of each marker in each subpopulation was heterogenous at the single-cell level (Figure 2E).

GrB is enriched in different subsets of Tregs in the periphery of kidney transplant recipients undergoing rejection. Next, we employed CyTOF to assess further whether the Tregs of allograft-rejecting patients had significantly higher expression of GrB compared with recipients with no evidence of rejection on biopsy. In a cohort of 20 allograft recipients undergoing biopsy for clinical indications, we found a significant increase 
A
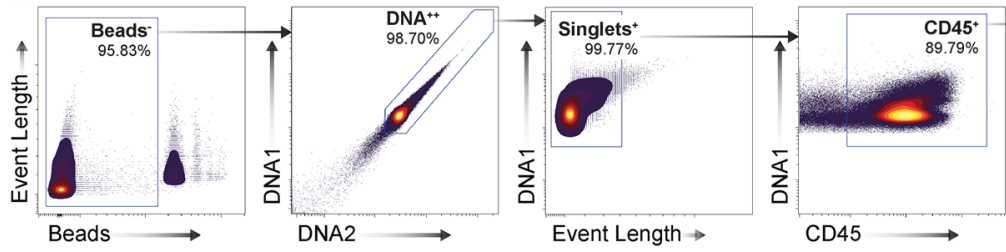

B

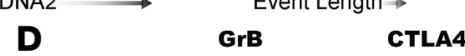

CD45 $\longrightarrow$
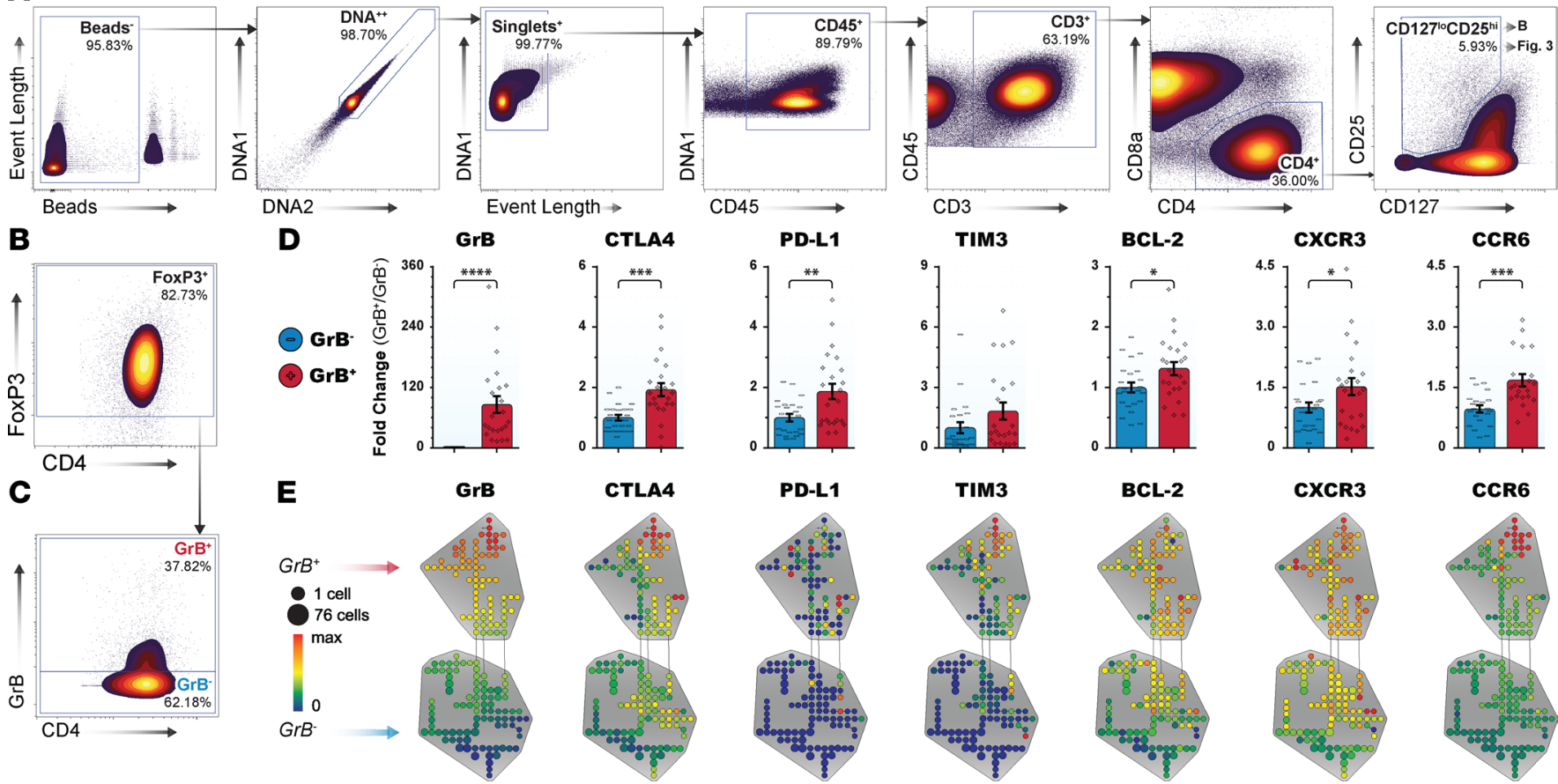

Figure 2. Characterization and phenotyping of $\mathrm{GrB}^{+}$and $\mathrm{GrB}^{-}$Tregs by various coinhibitory, costimulatory, and homing markers. (A) CyTOF gating strategy used to identify the (B) $\mathrm{CD}^{+} \mathrm{CD} 127^{\circ} \mathrm{CD} 25^{\mathrm{hi}} \mathrm{FoxP3} 3^{+}$cell population from the PBMCs of renal transplant patients to study the (C) $\mathrm{GrB}^{+}$and $\mathrm{CrB}^{-}$subsets. (D) Bar graphs show the fold change difference between the GrB subsets, based on their mean fluorescence intensity for the displayed markers. The primary GrB graph verifies that the chosen subsets are indeed $\mathrm{GrB}^{-}$and $\mathrm{GrB}^{+}$cells $\left(86.2 \pm 16.5,{ }^{* * *} P<0.0001\right)$, while the other graphs show that, among $\mathrm{GrB}{ }^{+}$Tregs, there is an increased expression of the coinhibitory markers CTLA4 (1.93 $\left.\pm 0.22,{ }^{* *} P<0.001\right)$, PD-L1 (1.87 $\left.\pm 0.26,{ }^{* *} P<0.01\right)$, TIM3 (1.83 \pm 0.43$)$, the anti-apoptotic BCL-2 (1.32 $\left.\pm 0.11,{ }^{*} P<0.05\right)$, and lastly the homing markers CXCR3 $\left(1.52 \pm 0.21,{ }^{*} P<0.05\right)$ and CCR6 $\left(1.68 \pm 0.15,{ }^{* * *} P<0.001\right)$. (E) Representative SPADE trees reiterate the notably increased activation state of $\mathrm{GrB}^{+}$Tregs compared with the $\mathrm{GrB}^{-}$counterpart. Despite the heterogeneity of expression patterns in the 2 subsets, $\mathrm{GrB}^{+}$Tregs demonstrate higher expression of coinhibitory, antiapoptotic, and homing markers. Each node represents a cell population, while the size of a node within the SPADE tree corresponds to the number of cells per population, and the node color indicates the signal intensity of the respective markers. BCL-2, B cell lymphoma 2; CCR6, C-C chemokine receptor 6; CTLA4, cytotoxic T-lymphocyte-associated protein 4; CXCR3, C-X-C chemokine receptor 3; FoxP3, forkhead box P3; GrB, granzyme B; PD-L1, programmed cell death protein 1; TIM3, T cell immunoglobulin and mucin-domain containing-3.

in the percentage of $\mathrm{GrB}^{+}$Tregs $\left(\mathrm{CD} 4^{+} \mathrm{CD} 25^{\text {high }} \mathrm{CD} 127^{\text {low }} \mathrm{FoxP}^{+}\right)$in the peripheral blood of allograft rejection patients compared with recipients with no evidence of rejection on biopsy (Figure 3, A-C). We then characterized the GrB expression in the different Treg populations. We observed that GrB expression was significantly increased in different subsets of Tregs, including resting Tregs $\left(C D 4^{+} \mathrm{CD} 25^{\text {high }} \mathrm{CD} 127^{\text {low }}\right.$ FoxP $3^{+} \mathrm{CD} 45 \mathrm{RA}^{\text {high }} \mathrm{CD}^{2} 5 \mathrm{RO}{ }^{\text {low }}$, Figure $\left.3, \mathrm{D}-\mathrm{F}\right)$ and memory Tregs $\left(\mathrm{CD} 4{ }^{+} \mathrm{CD} 25^{\text {high }} \mathrm{CD} 127^{\text {low }} \mathrm{FoxP}^{+} \mathrm{C}-\right.$ D45RA ${ }^{\text {low }} C D 45 R \mathrm{RO}^{\text {high }} \mathrm{BCL}-2^{\text {high }} \mathrm{Ki}-67^{\text {low }}$, Figure $3, \mathrm{G}-\mathrm{I}$ ), with a tendency toward significance in effector Tregs $\left(\mathrm{CD} 4^{+} \mathrm{CD} 25^{\text {high }} \mathrm{CD} 127^{\text {low }} \mathrm{FoxP} 3^{+} \mathrm{CD} 45 \mathrm{RA} \mathrm{A}^{\text {low }} \mathrm{CD} 45 \mathrm{RO} \mathrm{O}^{\text {high }} \mathrm{BCL}-2^{\text {low }} \mathrm{Ki}-67^{\text {high }}\right.$, Figure 3 , J and $\left.\mathrm{K}\right)$. Similarly, GrB expression was increased in Th1- and Th17-like Tregs, but this change did not reach statistical significance (Figure 3, L-Q). Possibly, this could be due to increased migration or increased apoptosis. We also looked at the expression of FoxP3 among the $\mathrm{GrB}^{+}$Tregs, and interestingly, there was more than a $50 \%$ increase in the mean fluorescence intensity of FoxP3 within the $\mathrm{GrB}^{+}$Tregs (Supplemental Figure 2; supplemental material available online with this article; https://doi.org/10.1172/jci.insight.91599DS1). To understand if GrB expression in Tregs is associated with increased Treg apoptosis, we performed flow cytometry analyses on the peripheral blood of patients undergoing kidney biopsy for clinical indication. The $\mathrm{GrB}^{+} \mathrm{CD}^{+} \mathrm{CD} 25^{\text {high }} \mathrm{FoxP}^{+}$Tregs of these patients showed markedly increased annexin $\mathrm{V}$ positivity, an indicator of apoptosis, compared with $\mathrm{GrB}^{-}$Tregs (Supplemental Figure 1).

In vitro-stimulated Tregs express GrB, GrA, and the serine PI9. To further investigate the role of $\mathrm{Gr}$ expression in activated Tregs, we examined Gr expression following in vitro stimulation of Tregs from healthy donor PBMCs. Immunomagnetically separated $\mathrm{CD} 4^{+} \mathrm{CD} 25^{\text {high }}$ Tregs from healthy volunteers were stimulated in vitro with anti-CD3 and anti-CD28 in the presence of a high concentration of IL-2 and analyzed by flow cytometry 72 hours later for CD4, CD25, CD127, Foxp3, GrB, and GrA (Figure 4, A and B). 
A


C

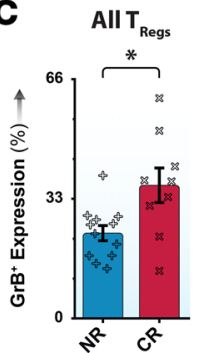

D
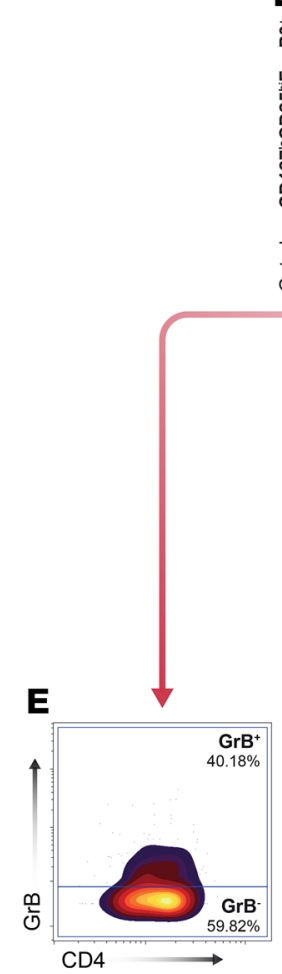

F Resting $\boldsymbol{T}_{\text {Regs }}$

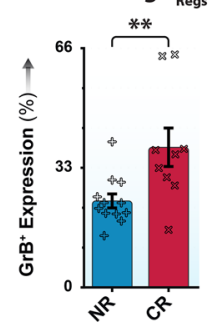

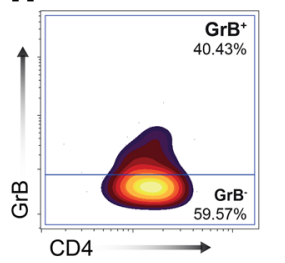


I Memory $\mathrm{T}_{\text {Regs }}$

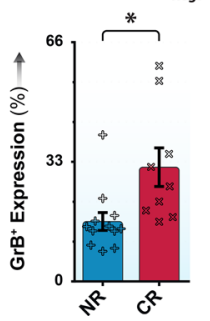

K Effector $T_{\text {Regs }}$

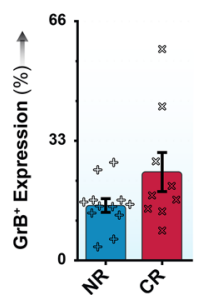

$\mathbf{L}$
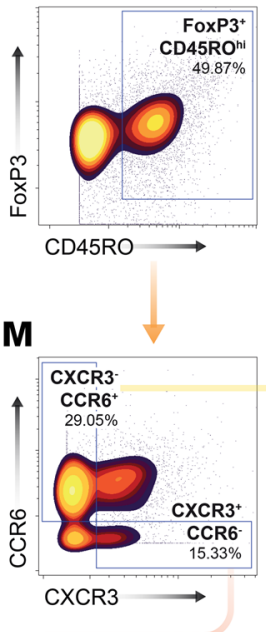

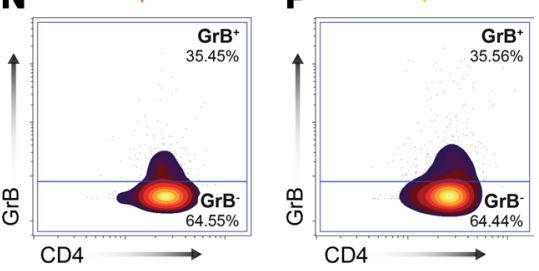

- $\mathrm{T}_{\mathrm{H}}$ 1-like $_{\text {Regs }}$

Q $\quad \mathrm{T}_{\mathrm{H}}$ 17-like $\mathrm{T}_{\text {Regs }}$
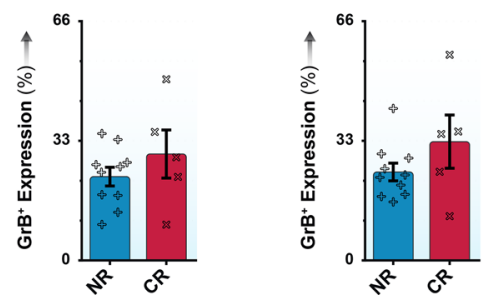

Figure 3. Quantitative differences between the expression of $\mathrm{GrB}^{+}$Tregs among clinically verified rejection and nonrejection kidney transplant patients. (A) Identification of the starting population of $C D 127^{\circ} \mathrm{CD} 25^{\mathrm{hi}}$ cells gated on $\mathrm{CD} 4^{+}$cells followed by the FoxP3 ${ }^{+}$gating. (B) Identification of the $\mathrm{CrB}^{+}$and $\mathrm{GrB}^{-}$subsets, and (C) the expression of $\mathrm{GrB}$ among all Tregs in patients classified as nonrejectors (NR) and cellular rejectors (CR), respectively $23.55 \% \pm 2.09 \%$ vs. $36.74 \% \pm 4.77 \%\left({ }^{*} P<0.05\right)$. (D) Divergence between resting Tregs and memory and effector Tregs, gated on the parent population of (A) $\mathrm{CD} 4{ }^{+} \mathrm{CD} 127^{\mathrm{lo}} \mathrm{CD} 25^{\mathrm{hi}} \mathrm{FoxP3}^{+}$Tregs. (E and $\mathbf{F}$ ) Identification of $\mathrm{GrB}$ subsets among the resting Tregs of NR and $\mathrm{CR}, 23.86 \% \pm 1.93 \%$ vs. $38.67 \% \pm$ $5.31 \%\left({ }^{*} P<0.01\right)$. (C) Divergence between memory and effector Tregs. (H and I) GrB subsets among the memory Tregs of NR and CR, $16.54 \% \pm 2.46 \%$ vs. $31.52 \% \pm 5.32 \%\left({ }^{*} P<0.05\right)$. (J and $\left.\mathbf{K}\right)$ GrB subsets among the effector Tregs of NR and CR, $15.17 \% \pm 1.89 \%$ vs. $24.39 \% \pm 5.38 \%$. (L) Identification of the FoxP3 ${ }^{+} C D 45 R 0^{\text {hi }}$ population gated on the parent population of $(\mathbf{A})$ of $C D 44^{+} C D 127^{10} C D 25^{\text {hi }}$ cells. $(\mathbf{M})$ Divergence between Th1-like and Th17-like Tregs. ( $\mathbf{N}$ and $\mathbf{O}$ ) GrB subsets among the Th1-like Tregs of NR and CR, $23.12 \% \pm 2.59 \%$ vs. $29.36 \% \pm 6.65 \%$. (P and $\mathbf{Q})$ GrB subsets among the Th17-like Tregs of NR and CR, $24.41 \% \pm 2.45 \%$ vs. $32.78 \% \pm 7.35 \%$. BCL-2, B cell lymphoma 2; CCR6, C-C chemokine receptor 6; CR, cellular rejector; CXCR3, C-X-C chemokine receptor 3; FoxP3, forkhead box P3; GrB, granzyme B; Ki-67, Kiel-67; NR, nonrejector; Th1, type $1 \mathrm{~T}$ helper cell; Th17, type 17 T helper cell.

Although only $9.1 \% \pm 2.5 \%$ of unstimulated Tregs were $\mathrm{GrB}^{+}$, a majority of the activated Tregs stained positively for $\operatorname{GrB}\left(60.4 \% \pm 4.1 \%,{ }^{* * * *} P<0.0001\right)$. Similarly, GrA levels increased from $9.8 \% \pm 2.5 \%$ to $65.6 \% \pm 3.1 \%\left({ }^{* * * *} P<0.0001\right)$. Spi6 is an endogenous murine serpin inhibitor of GrB and is a key homeostatic factor, regulating $\mathrm{GrB}$ activity and preventing $\mathrm{GrB}$ vesicular leakage $(13,27)$. The counterpart of Spi6 in human leukocytes is PI9. However, it is not known whether human Tregs upregulate PI9. We observed minimal expression of PI9 among unstimulated Tregs (4.9\% $\pm 1.7 \%)$; however, within 3 days of Treg activation, PI9 expression increased significantly to $67.7 \% \pm 0.8 \%$ as measured by flow cytometry ( ${ }^{* * * * P}$ $<0.0001$ ) (Figure $4 \mathrm{C}$ ) and by Western blot (Figure 4D). When non-Tregs $\left(C D 4^{+} \mathrm{CD} 25^{-}\right)$were activated in parallel, they did not upregulate PI9 in the same time frame (Figure 4D). Like the renal allograft-infiltrating $\mathrm{GrB}^{+}$Tregs (Figure 1), activated healthy donor peripheral blood $\mathrm{GrB}^{+}$Tregs expressed the apoptotic 
A
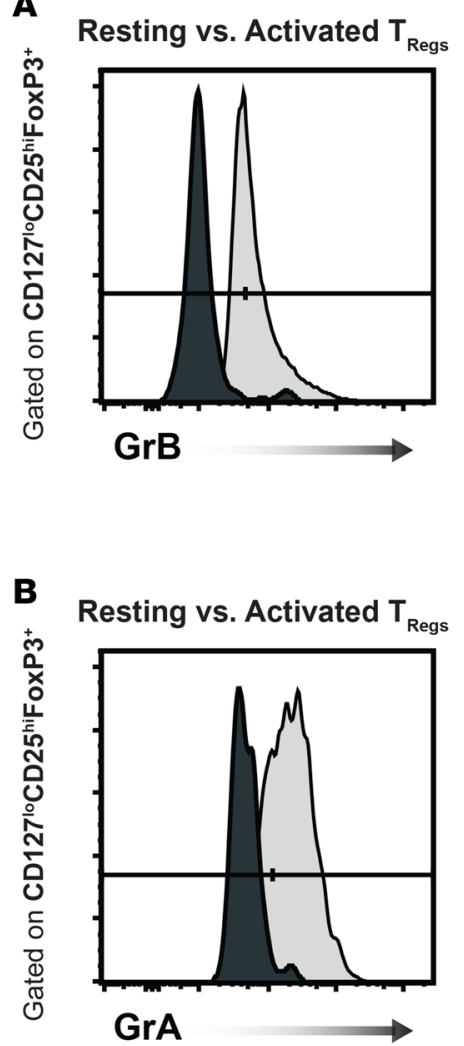



C
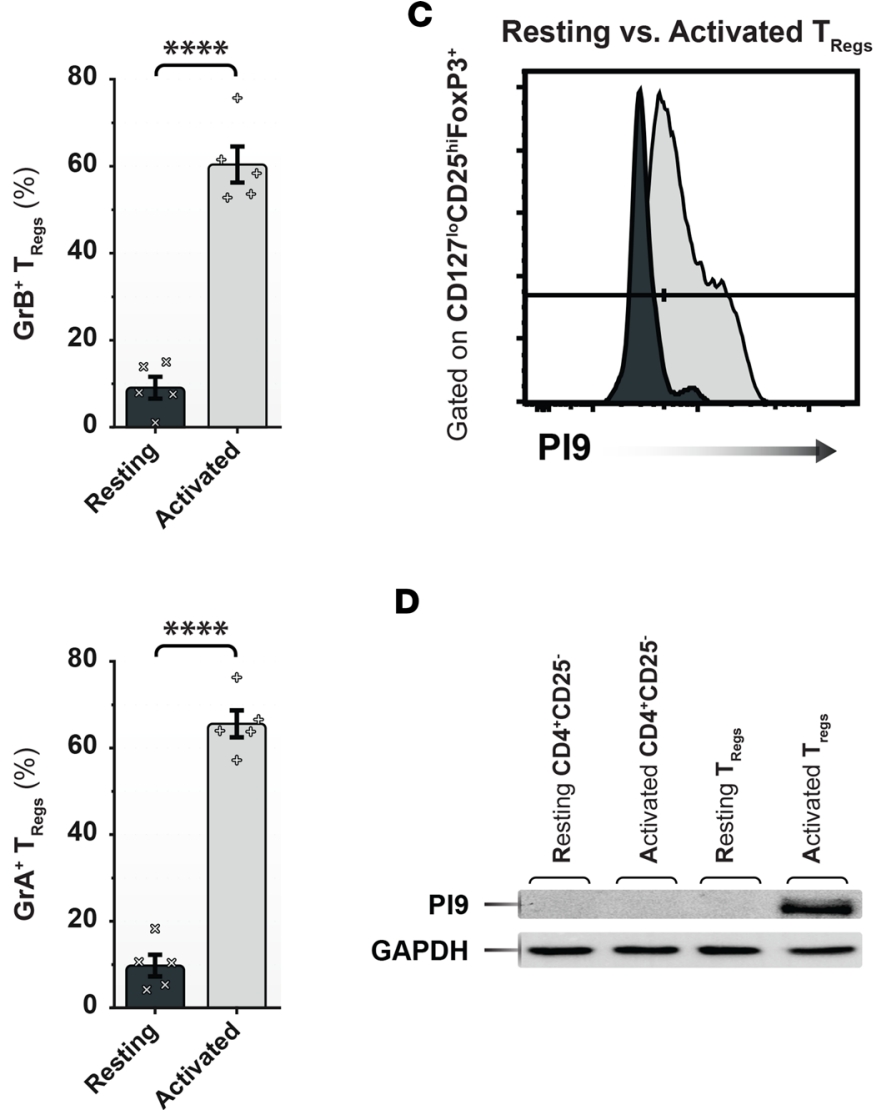

D

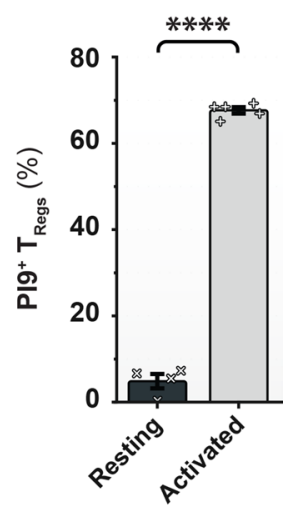

$\mathbf{E}$



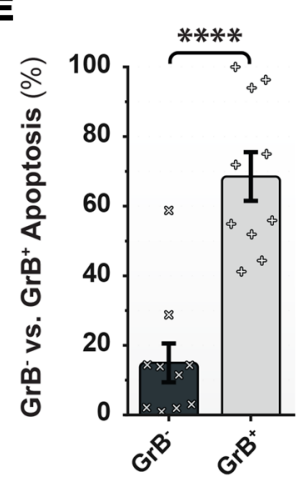

Figure 4. Increased expression of GrB, GrA, and the endogenous inhibitor of GrB, PI9, in in vitro stimulated human Tregs. Human Tregs (CD4+CD25 hi) were isolated from healthy individuals using magnetic beads and stimulated in vitro with anti-CD3/CD28 and IL-2 for 72 hours. (A) Representative example of flow cytometry analysis of $\mathrm{GrB}$ expression in resting and stimulated Tregs (gated on $\mathrm{CD} 127^{\circ} \mathrm{CD} 25^{\text {hi }}$ FoxP3 $3^{+}$). Bar graph shows the significant increase in GrB expression in Tregs between resting and stimulated Tregs ( $9.09 \% \pm 2.51 \%$ vs. $60.40 \% \pm 4.14 \%$, 3 separate experiments, $\left.{ }^{* * * *} P<0.0001\right)$. (B) Representative example of increased GrA expression between resting and stimulated Tregs $(9.76 \% \pm 2.50 \%$ vs. $65.58 \% \pm 3.10 \%, 3$ separate experiments, $\left.{ }^{* * * *} P<0.0001\right)$. (C) Representative example of increased PI9 expression between resting and stimulated Tregs $(4.86 \% \pm 1.66 \%$ vs. $67.66 \% \pm 0.75 \%, 2$ separate experiments, $\left.{ }^{* * *} P<0.0001\right)$. (D) Western blot analysis of cell lysates isolated from Tregs of healthy volunteers before and after stimulation, in addition to non-Treg CD4 cells before and after stimulation. Only activated Tregs showed expression of PI9. (E) Representative example of increased apoptosis between $\mathrm{GrB}^{-}$and $\mathrm{GrB}^{+}$Tregs $\left(14.98 \% \pm 5.58 \%\right.$ vs. 68.55\% $\pm 7.00 \%, 3$ separate experiments, $\left.{ }^{* * * *} P<0.0001\right)$. FoxP3, forkhead box P3; GrA, granzyme A; GrB, granzyme B; PI9, proteinase inhibitor 9.

annexin $\mathrm{V}$ marker at a much higher rate than the $\mathrm{GrB}^{-}$Tregs $\left(15.0 \% \pm 5.6 \%\right.$ vs. $68.6 \% \pm 7.0 \%$ for $\mathrm{GrB}^{-} \mathrm{vs}$. $\mathrm{GrB}^{+}$, respectively, ${ }^{* * * *} P<0.0001$ ) (Figure $4 \mathrm{E}$ ). Thus, healthy donor Tregs upregulate the Grs and undergo apoptosis within a few days of activation, despite their concurrent upregulation of the GrB serpin.

Grs leak from the granules of stimulated Tregs and are active. Gr-expressing cells are potentially susceptible to suicide by their own death-inducing proteases if the Grs leak out of their storage granules and are not inhibited. To examine whether Grs leak from cytotoxic granules in $\mathrm{GrB}^{+}$Tregs, we examined the subcellular localization of $\mathrm{GrB}$ and the lysosomal marker $\mathrm{CD} 107$, which also stains cytotoxic granules, in Tregs from healthy donors 2 days after in vitro stimulation (Figure 5, A-C). Although GrB and $\mathrm{CD} 107$ colocalized in some areas, $\mathrm{GrB}$ was also found both outside of the $\mathrm{CD} 107^{+}$granules in the cytosol and in the nucleus. Nuclear localization is a clear indication of $\mathrm{Gr}$ leakage from cytotoxic granules (released Grs concentrate in the nucleus of target cells) (36). We also studied the colocalization of $\mathrm{GrB}$ and its endogenous inhibitor PI9 upon activation by confocal microscopy. PI9 was mainly expressed in the cytoplasm of activated Tregs (Supplemental Figure 3A). Furthermore, flow cytometry analysis showed that most of the $\mathrm{GrB}^{+}$Tregs expressed PI9 after in vitro stimulation (Supplemental Figure 3B). We next compared GrA, GrB, and CD107 staining in healthy donor Tregs that were freshly isolated or were stimulated for 36 hours (Figure 5, D-H). There was little staining with either Gr in freshly isolated cells, and all the staining in resting cells was outside the nucleus. However, after activation, both Grs were highly expressed and could be found outside of $\mathrm{CD} 107^{+}$granules in 
A



B

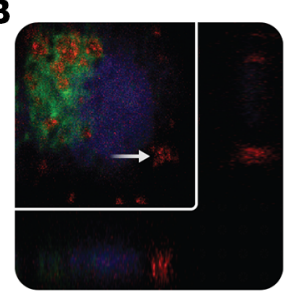

C

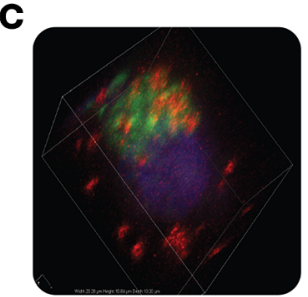

Dapi

GrB

CD107

D
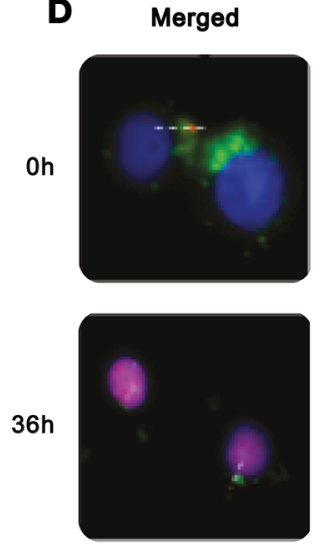

E
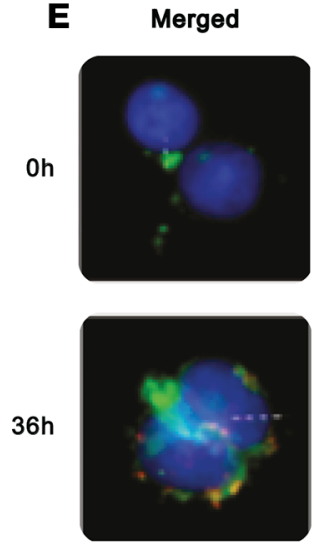

$\mathbf{F}$



CD107

$36 \mathrm{~h}$

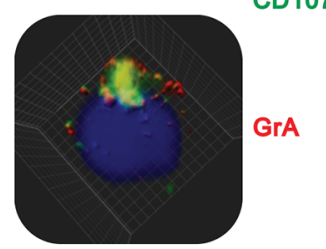

Dapi


Dapi
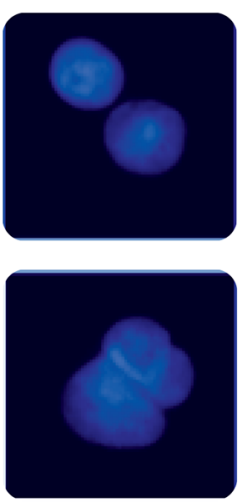

G



CD107

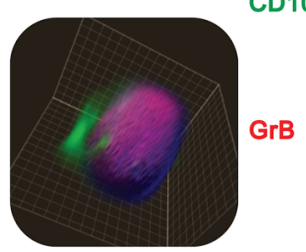

CD107


CD107
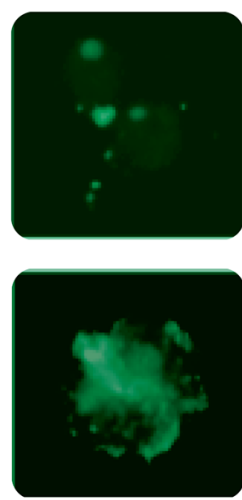
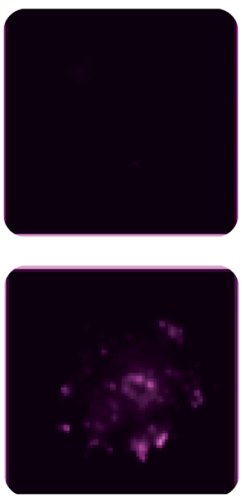

H

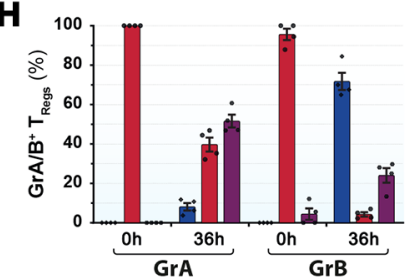

- Nucleus Cytoplasm Nucleus + Cytoplasm



Brightfield
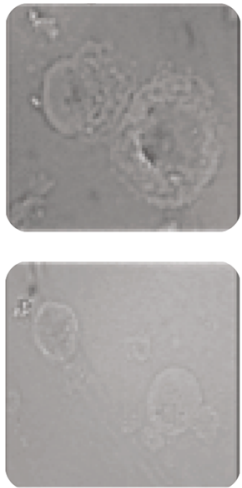

Brightfield


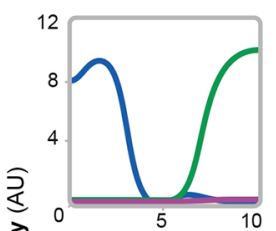

童



J

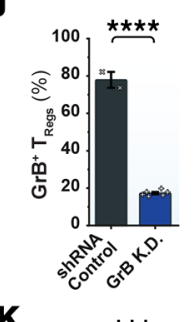

K


Figure 5. GrA and GrB leakage outside of the granules of activated Tregs and the induction of increased cytoplasmic granzyme activity. Isolated Tregs from the peripheral blood of healthy volunteers were stimulated in vitro for 48 hours with anti-CD3/CD28 and IL-2. Cells were stained with GrB (PE) and CD107 (FITC) and were fixed on a slide using a Cytospin centrifuge. They were analyzed by confocal microscopy as a Z stack. (A) Representative example of confocal microscopy $Z$ stack image of a stimulated Treg. Arrows in $\mathbf{A}$ show $\mathrm{GrB}^{+}$staining (PE) within the granule. (B) Arrows show GrB ${ }^{+}$staining (PE) outside 
the granule and within the cytoplasm. (C) Representative figure of 3-D microscopy image of stimulated Tregs that shows GrB presence outside the granules and within the cytoplasm of Tregs. (D) Representative figures of GrB localization by confocal microscopy. Channel intensities were measured along the dotted line to show localization of GrB (PE) with the nucleus (DAPI) or CD107 (FITC). (E) Representative figures of GrA localization by confocal microscopy. Channel intensities were measured along the dotted line to show localization of GrA (PE) with the nucleus (DAPI) or CD107 (FITC). (F) Three-dimensional volume view showing localization of GrA at 0 and 36 hours after stimulation. (C) Three-dimensional volume view showing localization of $\mathrm{GrB}$ at 0 and 36 hours after stimulation. (H) Graph shows the localization of GrA or GrB in Gr+ Tregs at 0 and 36 hours after in vitro stimulation. (I) Representative figure of flow cytometry analysis of a GranToxiLux assay measuring GrB activity in stimulated human Tregs, showing more than a 1-fold increase in intracellular granzyme activity in stimulated Tregs 1 day after stimulation (data represents 3 separate experiments, ${ }^{* *} P<0.01$ ). (J) Bar graph shows knockdown of GrB in Tregs isolated from healthy volunteers by shRNA-expressing lentiviruses to knock down GrB compared with shRNA control (data represents 1 of 2 separate experiments, $\left.{ }^{* * *} P<0.0001\right)$. (K) Graph shows significant reduction in apoptosis (annexin $V$ expression) of GrB knocked down Tregs compared with control (data represents 1 of 2 separate experiments, ${ }^{* * *} P<0.001$ ). AU, arbitrary unit; FL1, fluorescence channel (FL) 1; GrA, granzyme A; GrB, granzyme B; h, hour; K.D., knockdown; MFI, mean fluorescence intensity; PI9, proteinase inhibitor 9.

both the cytoplasm and nucleus (Supplemental Videos 1 and 2). In some cells, GrB stained exclusively in the nucleus, but this was uncommon for GrA. While GrB is inactive at the acidic $\mathrm{pH}$ of cytotoxic storage granules, it can be activated if it is released within the cell to the neutral $\mathrm{pH}$ conditions of the rest of the cell and is not inactivated by endogenous cellular protease inhibitors (28). To investigate whether Tregs have active GrB, we used the GranToxiLux assay (37) to compare GrB activity of healthy donor $\mathrm{CD} 4{ }^{+} \mathrm{CD} 25^{\text {high }}$ Tregs before and a day after stimulation with anti-CD3/CD28 and IL-2 (Figure 5I). GrB activity increased significantly after activation, suggesting that $\mathrm{GrB}$ is released from its storage granules and becomes activated within stimulated Tregs. Next, we reduced GrB production in activated Tregs using shRNA-expressing lentiviruses to see if GrB knockdown would protect Tregs from self-inflicted damage. Isolated Tregs from peripheral blood of healthy volunteers were infected with shRNA-expressing lentiviruses to knock down GrB and were then stimulated in vitro for 3 days. Knockdown of GrB expression decreased Treg apoptosis, as measured by flow cytometry (Figure 5, J and $\mathrm{K}$ ). These data suggest that GrB expression is not simply a correlate of Treg activation, but also contributes to Treg activation-induced cell death.

Gr substrates, including caspase 3, are cleaved in activated Tregs. GrB activates caspase 3 and also directly cleaves many key caspase substrates. GrA activates caspase-independent programmed cell death by cleaving a few of the same substrates (such as Parp1 and Lamin A/C) but mostly by cleaving an independent set of substrates, including SET and histones (38). To confirm that Grs are activated in stimulated Tregs, we used immunoblotting to probe for known Gr substrates before and at $0.5,1,2$, and 3 days after in vitro activation of healthy donor Tregs with anti-CD3/CD28 and IL-2 (Figure 6). Cells were cultured in the presence or absence of the pan-Gr inhibitor, 3,4-dichloroisocoumarin (DCI). Levels of full-length caspase 3; shared GrB, GrA, and caspase 3 substrates (Parp1, Lamin A/C); shared GrB and caspase 3 substrates (bid, $\alpha$-tubulin); and a unique GrA substrate (SET) were first seen to decline 2 days after stimulation, and most declined further still by day 3 , while levels of the loading control $\beta$-actin remained unchanged. In some cases, a cleavage fragment was visualized beginning on day 2. Moreover, cleavage of all these substrates was inhibited by DCI, indicating that the Grs were responsible for this cleavage. These data taken together show that the Grs are activated to cleave caspase 3 and cause apoptosis in stimulated Tregs, beginning around 2 days after stimulation.

Apoptosis of activated Tregs is partially mediated by Grs. When killer lymphocytes kill target cells, the Grs are taken up by mitochondria, where they generate ROS and disrupt the mitochondrial transmembrane potential $(39,40)$. Caspase activation similarly disrupts the mitochondrial transmembrane potential. To follow the course of cell death among healthy donor Tregs before and after stimulation, we used the mitochondrial dye $\mathrm{DiIC}_{1}$, which only fluoresces when the mitochondrial potential is intact and correspondingly decreases at the onset of apoptosis (Figure 7). DiIC $C_{1}$ staining in stimulated Tregs was reduced on days 2 and 3 following stimulation, confirming that stimulation caused Treg death. When these Tregs were restimulated on day 3 , the DiIC 1 staining was reduced further still by the next day, indicating that the restimulated cells that survived the first stimulation were prone to apoptosis. The leakage of Grs and activation of GrB in stimulated Tregs, which correlated with apoptosis, suggested that leaked Grs might be responsible for the apoptosis of activated Tregs. To assess whether Grs and/or caspases were responsible for Treg death, we added either DCI or the pan-caspase inhibitor ZVAD-fmk to the cell cultures. $\mathrm{DiIC}_{1}$ staining was significantly higher in the presence of either inhibitor. For the first stimulation, both inhibitors were equally effective at preventing death, suggesting that $\mathrm{GrB}$ activation of caspase 3 was an 


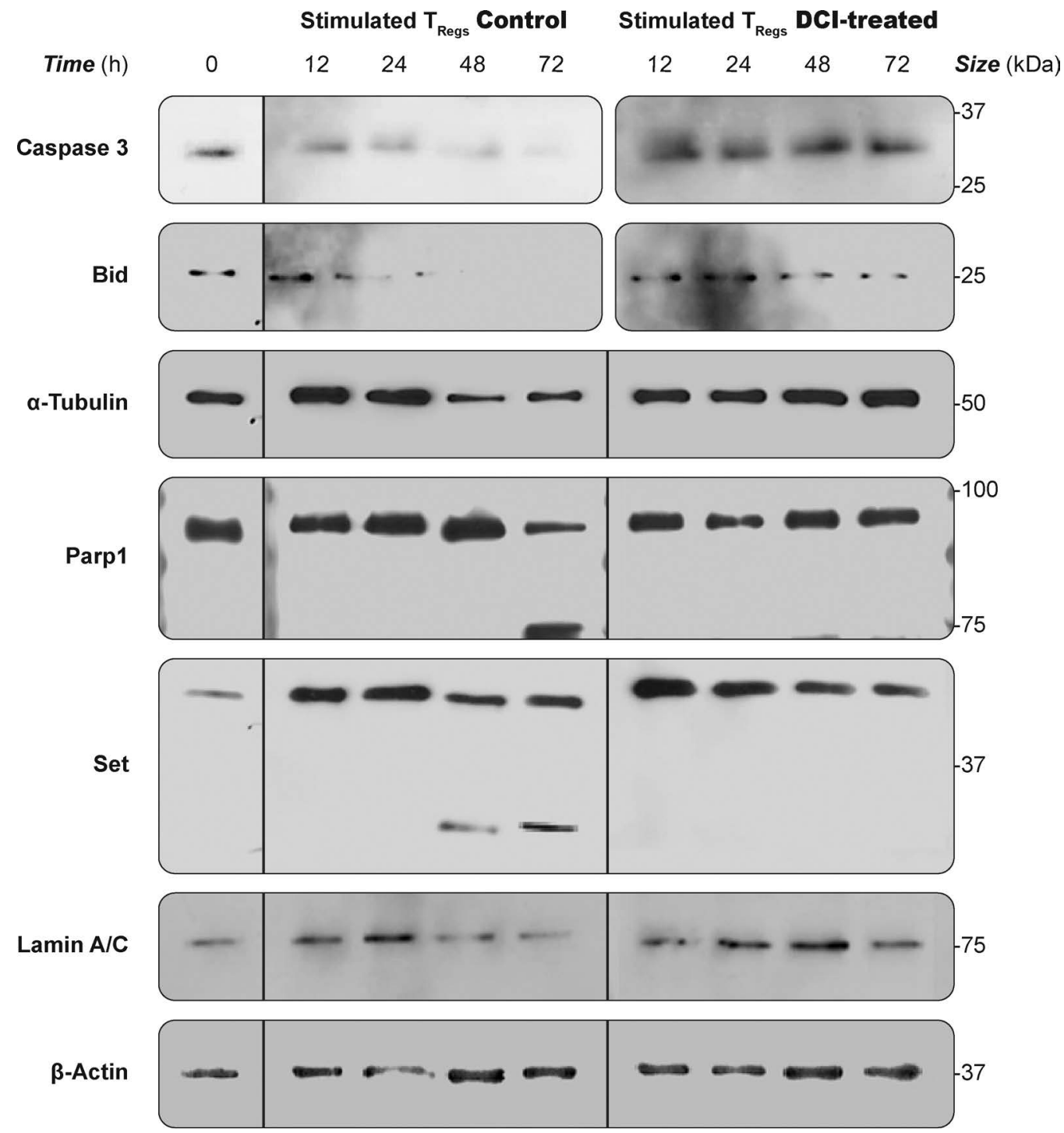

Figure 6. GrA and GrB cleavage of multiple mitochondrial, cytosolic, and nuclear proteins. Western blots of cell lysates from the same biological sample (i.e., stimulated Tregs) were contemporaneously run on different gels, showing different GrB and GrA targets in the cytoplasm, mitochondria, and nucleus. For blots with lysates on continuous lanes, the conditions have been separated by a thin black line, while noncontiguous lanes on different blots have been separated by white space. The Tregs were cultured for 3 days in the presence or absence of the pan-granzyme inhibitor DCI, and the lysates were harvested at $0.5,1,2$, and 3 days. $\beta$-Actin was used as the housekeeping protein. We observed that the cleavage of caspase 3 , Bid, and Lamin A/C were inhibited in the presence of DCl, reflecting the physiological role of the Grs in this cleavage. These findings affirm the role of the Grs in cleaving caspase 3 and in causing apoptosis in stimulated Tregs, notably within 2 days of stimulation. Bid, BH3 interacting-domain death agonist; DCl, 3,4-dichloro-isocoumarin; h, hour; kDa, kilodalton; Parp1, poly(ADP-ribose) polymerase 1.

inducer of cell death in stimulated Tregs. Upon restimulation, however, the protection afforded by DCI was significantly better than by ZVAD-fmk, suggesting that caspase-independent death by GrA and/or GrB became dominant. These data confirm that Treg death is Gr mediated.

Apoptosis of activated Tregs occurs without degranulation. Our confocal imaging results showing Gr staining outside cytotoxic granules in stimulated Tregs suggest that Tregs undergo suicide caused by Gr leakage from the safe storage within the cytotoxic granules. However, another possibility is death by fratricide caused by the uptake of Grs released during degranulation by neighboring Tregs. To examine this possibility, we stimulated Tregs in the presence of EDTA, which inhibits degranulation. Staining for cell surface CD107a, which localizes within cytosolic granules and is externalized during degranulation, confirmed inhibition of degranulation by EDTA (Supplemental Figure 4A). However, inhibiting degranulation did not affect apoptosis of $\mathrm{GrB}^{+}$ stimulated Tregs (Supplemental Figure 4B). Thus, cell death is mediated by autolysis caused by the intracellular leakage of Grs following Treg activation. 
A Day 3 - First Stimulation

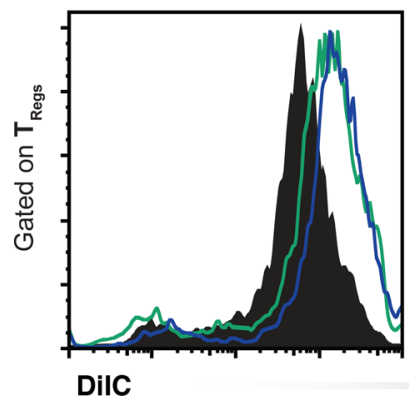

B Day 4 - After Re-Stimulation

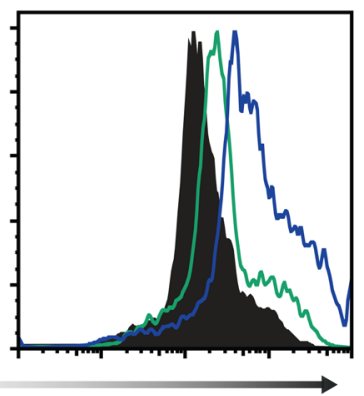

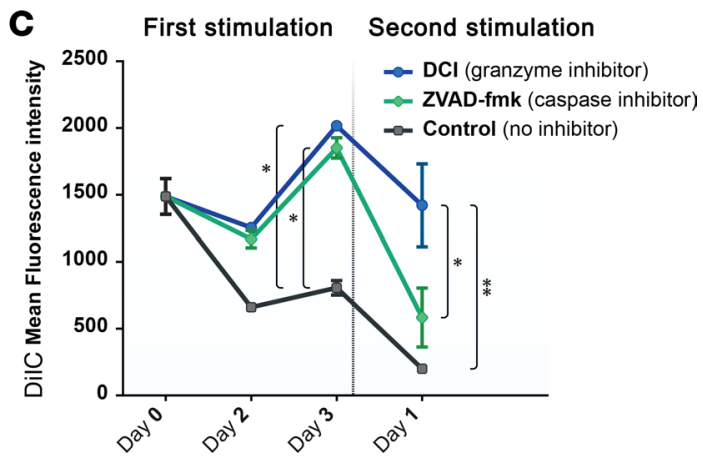

Figure 7. Granzyme B-mediated alteration of the mitochondrial inner membrane potential during in vivo Treg expansion. (A) Representative figure of flow cytometry analysis of Tregs stimulated in the presence or absence of DCI or ZVAD-fmk at day 3 after stimulation. Higher expression of DiIC is seen in the $\mathrm{DCl}$ and ZVAD-fmk treated groups compared with the untreated control. (B) Representative figure of Tregs restimulated (after an initial stimulation of 3 days) in the presence or absence of DCI or ZVAD-fmk at day 1 after restimulation. Higher expression of DiIC is seen in the DCI-treated group compared with the ZVAD-fmk-treated group, even more so compared with the untreated control. (C) Graph shows the MFI of DilC in stimulated Tregs over time. (Data represents 1 of 3 separate experiments, ${ }^{*} P<0.05$ and $\left.{ }^{* *} P<0.01\right)$. DCI, 3,4-dichloroisocoumarin; DilC, 1,1'-dimethyl-3,3,3',3'-tetramethylindodicarbocyanine iodide; ZVAD-fmk, carbobenzoxy-valyl-alanyl-aspartyl-[0-methyl]-fluoromethylketone.

\section{Discussion}

Here, we show that Tregs from patients undergoing renal graft rejection and in vitro-stimulated human Tregs from healthy donors highly express Grs and are prone to undergo apoptosis. We found that the Grs leak out of the cytotoxic granules, where they are stored in an inactive form, and become proteolytically active, activating caspase 3 and causing Gr-dependent apoptosis that is inhibited at least partially by a panGr inhibitor or by GrB knockdown. Stimulated Tregs undergo apoptosis during stimulation, despite the coinduction of the GrB inhibitor, the serpin PI9. The mouse homolog of PI9 (Spi6) was previously shown to protect both conventional $\mathrm{CD}^{+}$cytotoxic $\mathrm{T}$ cells and mouse Tregs from self-inflicted death by any stray Grs that leak out of cytotoxic granules $(13,41-43)$, but it is clearly not adequate to completely protect stimulated human Tregs from apoptosis. Our data suggest that $\mathrm{GrB}$ is not only induced by Treg activation during kidney transplant rejection, but also plays a role in the activation-induced cell death of Tregs, dampening the controls that suppress rejection.

Why PI9 is not effective in protecting Tregs from cell death is a question that requires further study. Does the level of GrB in stimulated Tregs overwhelm the level of PI9, which irreversibly binds to target Grs in a 1:1 ratio? Released Grs concentrating in the nucleus may give an indication that leaked Gr indeed overwhelm the endogenous inhibitor PI9 present in the cytoplasm. Additionally, there are no known intracellular serpins for the other major Gr, GrA. Is GrA a major mediator of Treg death?

Increased apoptosis of activated Tregs may be a key element in restoring normal immune homeostasis. During the late stage of an active immune response, Tregs proliferate, traffic, and accumulate at the site of inflammation to suppress the effector response (44). However, the increased apoptosis of activated Tregs may explain the subsequent contraction of the Treg population and full restoration of immune homeostasis. The decline in Tregs should allow antigen-specific T cell clones to be reactivated in case of reexposure to the same antigen.

Tregs express Grs as well as perforin and can kill antigen-specific effector CD4 and CD8 T cells in a Gr-dependent, cell contact-dependent manner by granular release. Our group and others have reported a central role of murine Treg-secreted GrB in inducing effector cell suppression and allograft tolerance $(11,13,14)$. However, the importance of direct killing in the immunosuppressive function of Tregs compared with other mechanisms, including depletion of IL-2, is unclear. If Grs both promote Treg function by direct killing of activated effector cells and also are responsible for limiting the survival of activated Tregs, then KO of Grs or perforin could either promote or inhibit Treg function, depending on the strength of each effect. Therefore, conclusions about the importance of direct cytotoxicity in Treg function based on genetically deficient mouse strains need to be reexamined in light of our findings here.

Our finding of self-inflicted damage of stimulated Tregs, which is enhanced by restimulation (Figure 7), has important implications for transplantation. Continuous stimulation of Tregs in vivo during autoimmune disease or after transplantation may lead to their death. Cellular therapy using ex vivo- 
expanded Tregs is a promising approach for treating immune-mediated diseases, such as allograft rejection, caused by an overexuberant immune response. Although multiple clinical trials of Treg therapy have been undertaken, the results of these published trials have been suboptimal. Brunstein and colleagues showed that transferred Tregs peaked in the periphery 2 days after transfer and decreased to baseline by day 14 (5). Two days is the time at which we first observed in vitro Gr leakage, activation, and apoptosis after restimulation. Similarly, numbers of peripheral Tregs returned to pretransfer levels between week 2 and 8 after transfer in patients with type 1 diabetes treated with Treg transfer therapy (6). The mechanism underlying the loss of adoptively transferred Tregs has not been described and yet is central to understanding the fate of these cells in vivo. Although Bluestone and colleagues recently showed that Tregs could persist in the blood for months after adoptive transfer, the number of persistent cells a few days after the transfer was less than $20 \%$ of the peak level (20). It is important to note that the low level of circulating Tregs in clinical studies could either be due to Treg death or to their migration from the blood.

Large numbers of Tregs are required to induce tolerance in models of solid organ transplantation, GvHD, or type 1 diabetes, thereby necessitating efficient and repeated in vitro stimulation to expand Treg clones $(9,45-48)$. Given their limited in vivo survival and the necessity for ex vivo expansion for clinical applications, a detailed understanding of the mechanisms of Treg survival and homeostasis will be critical to adopt this approach successfully. Changing the in vitro expansion conditions or coadministering specific immune modulators that prolong Treg survival to patients receiving ex vivoexpanded Treg therapy may improve the persistence of transferred cells, based on a better understanding of the factors that influence stimulated Treg death. More successful Treg expansion protocols have been achieved recently by adding rapamycin to the culture (16-19). Interestingly, production of $\mathrm{GrB}$ in Tregs can be suppressed by inhibitors of the PI3K/mTOR pathway, such as rapamycin (49), which may explain the beneficial effect of mTOR inhibitors on ex vivo expansion of Tregs (50). However, the $\mathrm{PI} 3 \mathrm{~K} / \mathrm{mTOR}$ pathway is also critical for Treg survival (51), which is why alternative approaches may be needed to reduce Gr leakage or activity within the cytoplasm of Tregs without suppressing the role of Grs in Treg function.

While human Tregs have previously been shown to produce GrB upon activation in vitro, here, we describe the accumulation of GrB-expressing Tregs within rejecting kidney allografts. Furthermore, Tregs from the peripheral blood of kidney transplant recipients undergoing rejection have higher GrB expression than Tregs from nonrejecting control patients. Suthanthiran and colleagues showed a correlation between FoxP3 and GrB mRNA isolated from urinary cells during acute cellular rejection in kidney transplant recipients $(52,53)$. These data suggest a state of increased Treg activation during allograft rejection. Our CyTOF analysis comparing protein expression in peripheral blood shows that GrB-expressing Tregs are indeed more activated than non-GrB expressing Tregs, as reflected by their increased expression of checkpoint inhibitors and costimulatory molecules. Elevated expression of coinhibitory molecules is associated with increased suppressive Treg activity against effector cells (54-57). Furthermore, peripheral $\mathrm{GrB}^{+}$Tregs also had elevated expression of chemokine receptors (CXCR3 and CCR6) essential for Th1- and Th17-like Treg homing to areas of inflammation (58-60). Th1 and Th17 have been implicated in kidney transplant rejection in humans $(61,62)$. Th1- and Th17like Tregs have recently been described to home to areas of inflammation mediated by Th1 and Th17 conventional T cells, respectively, to suppress their function (63). Our data show that during kidney transplant rejection, Tregs get activated, express Grs, and increase their expression of the homing receptors CXCR3 (in Th1-like Tregs) and CCR6 (in Th17-like Tregs), which will cause them to home to the areas of rejection mediated by Th1 and Th17 cells. While GrB expression was increased in Th1- and Th17-like Tregs, it did not reach statistical significance (Figure 3, N-Q). This might be explained by migration of those Tregs from the blood to the area of rejection. However, the ratio of Tregs expressing GrB is relatively high to be explained only by the activation of allospecific Tregs. This means that some of the Treg activation we measured might be nonspecific and due to the proinflammatory state that accompanies rejection.

These data improve our understanding of Treg homeostasis during transplant rejection and raise the therapeutic possibility that manipulating Gr leakage could be used to design more effective Treg therapies. Unfortunately, we are not aware of a way to selectively do that. 


\section{Methods}

Immunofluorescence. Paraffin-fixed slides from kidney transplant biopsies showing rejection were stained using immunofluorescent antibodies against FoxP3 (FITC, 3G3) and GrB (ab4059) (Abcam).

Isolation of peripheral blood mononuclear cells (PBMC). Peripheral blood samples, obtained from kidney transplant recipients at the time of a kidney biopsy for deteriorating kidney function, were collected between 7-9 a.m. and were processed within 4 hours at the Immunological Core Facility at the Transplant Research Center, Brigham and Women's Hospital. Blood samples from healthy volunteers were obtained after informed, signed consent. PBMCs were isolated by density gradient centrifugation at 1,000 $\mathrm{g}$ for 30 minutes at $20^{\circ} \mathrm{C}$ using Lymphoprep density gradient medium (Stemcell Technologies).

Peripheral blood analysis of Tregs by CyTOF. Cells were first stained with MaxPar Intercalator-Rh (Fluidigm Sciences Inc.) to identify viable cells, and then human Fc Receptor Blocking Reagent (Miltenyi Biotec Inc.) was added. The cells were stained with cell surface antibodies against CD45 (HI30, 89Y), CD3 (UCHT1, 115Ln), CD8a (RPA-T8, 146Nd), CD4 (RPA-T4, 145Nd), CD25 (2A3, 169Tm), CD127 (A019D5, 168Er), CTLA4 (L3D10, 170Er), PD-L1 (29E.2A3, 148Nd), TIM3 (F38-2E2, 153Eu), CXCR3 (G025H7, 163Dy), CCR6 (G034E3, 141Pr), CD45RO (UCHL1, 164Dy), and CD45RA (HI100, 155Gd) purchased from Fluidigm Sciences Inc. Afterward, they were washed with MaxPar Cell Staining Buffer (Fluidigm Sciences Inc.) and fixed and permeabilized with fixation/permeabilization buffer (Fluidigm Sciences Inc., 201067). Cells were then stained with intracellular antibodies against FoxP3 (PCH101, 165Ho), GrB (GB11, 167Er), Ki-67 (Ki67, 161Dy), and BCL-2 (100, 152Sm) produced by the DF/HCC antibody core facility and washed with Cell Staining Buffer containing 0.3\% saponin. For mass cytometry analysis, cells were stained with MaxPar Ir DNA Intercalator (Fluidigm Sciences Inc.) overnight at $4^{\circ} \mathrm{C}$. Cells were then washed with Cell Staining Buffer and with MaxPar Water (Fluidigm Sciences Inc.). Cells were analyzed on a Helios mass cytometer (Fluidigm Sciences Inc.) using Cytobank software (Cytobank Inc.).

Human Treg isolation and stimulation. $\mathrm{CD}^{+} \mathrm{CD} 25^{+} \mathrm{T}$ cells, isolated from freshly separated PBMCs using MACS magnetic beads (Miltenyi Biotec Inc.), were cultured in complete medium RPMI (BioWhittaker Classic Cell Culture Media, Lonza Biologics Inc.) with 10\% pooled human AB serum (GemCell U.S. Origin Human Serum AB), 1\% L-glutamine, and 1\% penicillin-streptomycin, supplemented with $1000 \mathrm{IU} /$ $\mathrm{ml}$ of recombinant methionyl human IL-2 (Amgen). Functional grade purified anti-human CD3 (OKT3) and functional grade purified anti-human CD28 (CD28.2) (eBioscience) were added at $2 \mu \mathrm{g} / \mathrm{ml}$. In some experiments, 5-100 $\mu \mathrm{M}$ Gr inhibitor 3,4-Dichloroisocoumarin (DCI) (Santa Cruz Biotechnology Inc.) or $20 \mu \mathrm{M}$ Caspase inhibitor ZVAD-FMK (V116) (MilliporeSigma) were added as indicated.

shRNA lentiviral particle preparation. Bacterial glycerol stocks were obtained from the MilliporeSigma shRNA bank (GrB, TRCN0000372388). Afterward, plasmids containing the target shRNA were extracted using an Endotoxin Free Plasmid Maxiprep kit (Qiagen). On day 1, 293T cells were transfected using Lipofectamine 2000 (Thermo Fisher Scientific) along with envelope and packaging plasmids (Vpx cDNA, p6mut8.9, and pHEF-VSVG) in a concentration of $1 \mu \mathrm{g} / \mathrm{ml}$. On day 2, the culture medium was replaced by X-VIVO 20 (Lonza BioWhittaker) medium; the lentiviruses were harvested 48 hours later and centrifuged at $400 \mathrm{~g}$ for 5 minutes at $4^{\circ} \mathrm{C}$. The final product was filter sterilized using a $0.45-\mu \mathrm{m}$ syringe filter (EMD Millipore).

Treg transfection. PBMCs were isolated from peripheral blood of healthy individuals using a SepMate tube (Stemcell Technologies) optimized protocol. Tregs were isolated using an EasySep $\mathrm{CD} 4{ }^{+} \mathrm{CD} 127^{\text {low }} \mathrm{CD} 25^{+}$isolation protocol (Stemcell Technologies) and stimulated as outlined above. One day after stimulation, Tregs were infected by adding the viruses in a 1:1 ratio with $8 \mu \mathrm{g} / \mathrm{ml}$ polybrene (Santa Cruz Biotechnology Inc.), and then restimulated with anti-CD3/CD28 and IL-2. Spinoculation was performed at $300 \mathrm{~g}$ for 1 hour at $4^{\circ} \mathrm{C}$, and the Tregs were again cultured for 4 days. On day 4 , the Tregs were treated with puromycin $(1.5 \mu \mathrm{g} / \mathrm{ml})$, restimulated with IL-2, and cultured for a final 24 hours before flow cytometry staining.

Flow cytometry. Samples were analyzed using a FACS Canto-II flow cytometer (BD Biosciences) and FlowJo software version 9.3.2 (TreeStar). Antibodies to human CD4 (RPA-T4), CD25 (M-A251), and CD127 (A019D5) and annexin V (all from BioLegend) were purchased. For intracellular staining, permeabilization solution, and antibodies to human FoxP3 (236A/E7) (Thermo Fisher Scientific); GrA (CB9) and

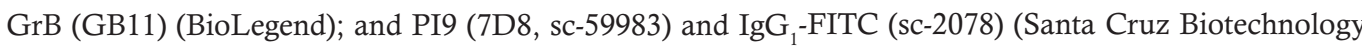
Inc.) were used following the manufacturer's protocol. For the apoptosis analysis of DCI-treated samples, data were normalized to unstimulated Tregs cultured for 12 hours. 
Western blot. Samples were lysed in RIPA buffer (Thermo Fisher Scientific) using a protease inhibitor mixture (Roche Diagnostics). Protein levels were analyzed by a BCA kit (Pierce Biotechnology Inc.), and $20 \mu \mathrm{g}$ total protein was loaded per lane for electrophoresis through $10 \%$ SDS page gels and transfer to PVDF membranes. Blots were blocked for 1 hour with TBS-Tween 20 containing 5\% powdered skim milk and then probed overnight at $4^{\circ} \mathrm{C}$ with rabbit polyclonal anti-human PI9 (Santa Cruz Biotechnology Inc., 7D8), rabbit anti-human caspase 3 (Cell Signaling Technology, 9662S), mouse anti-human FoxP3 monoclonal (Abcam, 236A/E7), rabbit anti-human Lamin A/C polyclonal (Cell Signaling Technology, 2032S), rabbit anti-human $\beta$-actin polyclonal (Abcam, ab8227), mouse antihuman $\alpha$-tubulin monoclonal (MilliporeSigma, MABT205), mouse anti-human Parp1 monoclonal (Santa Cruz Biotechnology Inc., sc-136208), and rabbit anti-human Bid polyclonal (Abcam, ab62469). The SET antibody was produced in rabbits using the synthetic peptide C-Ahx-AQAAKVSKKELN$\mathrm{SN}$-amide by the DF/HCC antibody core facility. The antibody was purified from the rabbit serum using full-length SET protein bound to Affi-gel beads (Bio-Rad) and eluted using $100 \mathrm{mM}$ Glycine $\mathrm{pH}$ 3 into $2 \mathrm{M}$ Tris at $\mathrm{pH}$ 7.5. After washing, blots were probed for 1 hour with the appropriate HRP-conjugated secondary antibodies and developed using Immobilon Western Chemiluminescent HRP substrate (EMD Millipore).

GranToxiLux assay. Gr activity after Treg in vitro stimulation was compared with that of unstimulated Tregs by flow cytometry using the GranToxiLux kit (GranToxiLux, OncoImmunin Inc.) following the manufacturer's protocol.

Confocal microscopy. Isolated human Tregs from healthy donors were stimulated for 72 hours using plate-bound anti-human CD3/CD28 in the presence of IL-2 as described above. Cytospun samples were stained with FITC anti-human CD107a (H4A3), and Alexa Fluor 594 anti-human GrA (CB9) (both from BioLegend), as well as PE mouse anti-human GrB (GB11) (BD Pharmingen), and PI9 (7D8) (Santa Cruz Biotechnology Inc.) following the manufacturer's recommendations. Slides were mounted with VectaShield with DAPI (Vector Labs) and imaged by confocal microscopy using an inverted, fully motorized Axio Observer spinning disk confocal microscope (Carl Zeiss Microimaging Inc.) equipped with a cooled electron multiplication CCD camera with $512 \times 512$ resolution (QuantEM, Photometics). Images were analyzed using SlideBook V5.0 (Intelligent Imaging Inc.) software. Three-dimensional image stacks were obtained along the $\mathrm{z}$-axis using the $63 \times$ oil immersion objective by acquiring sequential optical planes spaced $0.25 \mu \mathrm{m}$ apart. Raw images were deconvolved using SlideBook.

Statistics. Two-sided, unpaired Student's $t$ tests were used to compare 2 independent groups. $P \leq 0.05$ was considered statistically significant. Statistical analyses were performed using GraphPad Prism version 6.00 (GraphPad Software). Data represent means \pm SEM.

Study approval. Kidney transplant recipients admitted for clinically indicated kidney transplant biopsy at the Brigham and Women's Hospital were recruited after obtaining informed, signed consent. The study protocol was reviewed and approved by an Institutional Review Board at the Brigham and Women's Hospital and was conducted in full conformance with the principles of the Declaration of Helsinki.

\section{Author contributions}

ATK, ESK, PL, SKE, and SKR designed and performed experiments, analyzed and interpreted data, and drafted the manuscript. AA, ABM, AR, FD, JPA, KRP, OM, and TJB performed experiments and analyzed data. AC, FD, IG, JL, LVR, MM, RA, and WE helped with the study design. JL critically revised the manuscript. JA designed the study, interpreted the data, and critically revised and finalized the manuscript.

\section{Acknowledgments}

This work is supported by an American-Heart-Association grant (13FTF17000018 to JA), the Innovative Basic Science grant from the American Diabetes Association (1-17-IBS-206 to JA), and a K24 grant (AI116925 to RA).

Address correspondence to: Jamil Azzi, Transplant Research Center, Brigham and Women's Hospital, 221 Longwood Avenue, Boston, Massachusetts 02115, USA. Phone: 617.525.7359; Email: jazzi@rics.bwh.harvard.edu. 
1. Nomura T, Sakaguchi S. Foxp3 and Aire in thymus-generated Treg cells: a link in self-tolerance. Nat Immunol. 2007;8(4):333-334.

2. Wing K, Sakaguchi S. Regulatory T cells exert checks and balances on self tolerance and autoimmunity. Nat Immunol. 2010;11(1):7-13.

3. Wieckiewicz J, Goto R, Wood KJ. T regulatory cells and the control of alloimmunity: from characterisation to clinical application. Curr Opin Immunol. 2010;22(5):662-668.

4. Burrell BE, Nakayama Y, Xu J, Brinkman CC, Bromberg JS. Regulatory T cell induction, migration, and function in transplantation. J Immunol. 2012;189(10):4705-4711.

5. Brunstein CG, et al. Infusion of ex vivo expanded T regulatory cells in adults transplanted with umbilical cord blood: safety profile and detection kinetics. Blood. 2011;117(3):1061-1070.

6. Marek-Trzonkowska N, et al. Administration of CD4+CD25highCD127-regulatory T cells preserves $\beta$-cell function in type 1 diabetes in children. Diabetes Care. 2012;35(9):1817-1820.

7. Matsuoka K, et al. Low-dose interleukin-2 therapy restores regulatory T cell homeostasis in patients with chronic graft-versushost disease. Sci Transl Med. 2013;5(179):179ra43

8. Riley JL, June $\mathrm{CH}$, Blazar BR. Human T regulatory cell therapy: take a billion or so and call me in the morning. Immunity. 2009;30(5):656-665.

9. Trzonkowski P, et al. First-in-man clinical results of the treatment of patients with graft versus host disease with human ex vivo expanded CD4+CD25+CD127- T regulatory cells. Clin Immunol. 2009;133(1):22-26.

10. Walsh PT, Taylor DK, Turka LA. Tregs and transplantation tolerance. J Clin Invest. 2004;114(10):1398-1403.

11. Cao X, et al. Granzyme B and perforin are important for regulatory T cell-mediated suppression of tumor clearance. Immunity. 2007;27(4):635-646

12. Vignali DA, Collison LW, Workman CJ. How regulatory T cells work. Nat Rev Immunol. 2008;8(7):523-532.

13. Azzi J, et al. Serine protease inhibitor 6 plays a critical role in protecting murine granzyme B-producing regulatory $\mathrm{T}$ cells. J Immunol. 2013;191(5):2319-2327.

14. Gondek DC, Lu LF, Quezada SA, Sakaguchi S, Noelle RJ. Cutting edge: contact-mediated suppression by CD4+CD25+ regulatory cells involves a granzyme B-dependent, perforin-independent mechanism. J Immunol. 2005;174(4):1783-1786.

15. Singh K, et al. Superiority of rapamycin over tacrolimus in preserving nonhuman primate Treg half-life and phenotype after adoptive transfer. Am J Transplant. 2014;14(12):2691-2703.

16. Battaglia M, Stabilini A, Migliavacca B, Horejs-Hoeck J, Kaupper T, Roncarolo MG. Rapamycin promotes expansion of functional CD4+CD25+FOXP3 + regulatory $\mathrm{T}$ cells of both healthy subjects and type 1 diabetic patients. J Immunol. 2006;177(12):8338-8347.

17. Hippen KL, et al. Massive ex vivo expansion of human natural regulatory $\mathrm{T}$ cells (T(regs)) with minimal loss of in vivo functional activity. Sci Transl Med. 2011;3(83):83ra41.

18. Battaglia M, Stabilini A, Tresoldi E. Expanding human T regulatory cells with the mTOR-inhibitor rapamycin. Methods Mol Biol. 2012;821:279-293.

19. Canavan JB, et al. Developing in vitro expanded CD45RA+ regulatory T cells as an adoptive cell therapy for Crohn's disease. Gut. 2016;65(4):584-594.

20. Bluestone JA, et al. Type 1 diabetes immunotherapy using polyclonal regulatory T cells. Sci Transl Med. 2015;7(315):315ra189.

21. Grossman WJ, Verbsky JW, Barchet W, Colonna M, Atkinson JP, Ley TJ. Human T regulatory cells can use the perforin path way to cause autologous target cell death. Immunity. 2004;21(4):589-601.

22. Grossman WJ, Verbsky JW, Tollefsen BL, Kemper C, Atkinson JP, Ley TJ. Differential expression of granzymes A and B in human cytotoxic lymphocyte subsets and T regulatory cells. Blood. 2004;104(9):2840-2848.

23. Lafont V, et al. Plasticity of $\gamma \delta$ T Cells: Impact on the Anti-Tumor Response. Front Immunol. 2014;5:622.

24. Ashton-Rickardt PG. An emerging role for Serine Protease Inhibitors in T lymphocyte immunity and beyond. Immunol Lett. 2013;152(1):65-76.

25. Silverman GA, et al. The serpins are an expanding superfamily of structurally similar but functionally diverse proteins. Evolution, mechanism of inhibition, novel functions, and a revised nomenclature. J Biol Chem. 2001;276(36):33293-33296.

26. Lovo E, Zhang M, Wang L, Ashton-Rickardt PG. Serine protease inhibitor 6 is required to protect dendritic cells from the kiss of death. J Immunol. 2012;188(3):1057-1063.

27. Zhang M, et al. Serine protease inhibitor 6 protects cytotoxic T cells from self-inflicted injury by ensuring the integrity of cytotoxic granules. Immunity. 2006;24(4):451-461.

28. Chowdhury D, Lieberman J. Death by a thousand cuts: granzyme pathways of programmed cell death. Annu Rev Immunol. 2008;26:389-420.

29. Duhen T, Duhen R, Lanzavecchia A, Sallusto F, Campbell DJ. Functionally distinct subsets of human FOXP3+ Treg cells that phenotypically mirror effector Th cells. Blood. 2012;119(19):4430-4440.

30. Kleinewietfeld M, Puentes F, Borsellino G, Battistini L, Rötzschke O, Falk K. CCR6 expression defines regulatory effector/ memory-like cells within the CD25(+)CD4+ T-cell subset. Blood. 2005;105(7):2877-2886.

31. Szanya V, Ermann J, Taylor C, Holness C, Fathman CG. The subpopulation of CD4+CD25+ splenocytes that delays adoptive transfer of diabetes expresses L-selectin and high levels of CCR7. J Immunol. 2002;169(5):2461-2465.

32. Hoerning A, et al. Subsets of human CD4(+) regulatory T cells express the peripheral homing receptor CXCR3. Eur J Immunol. 2011;41(8):2291-2302.

33. Hockenbery DM, Oltvai ZN, Yin XM, Milliman CL, Korsmeyer SJ. Bcl-2 functions in an antioxidant pathway to prevent apoptosis. Cell. 1993;75(2):241-251.

34. Pinkoski MJ, et al. Granzyme B-mediated apoptosis proceeds predominantly through a Bcl-2-inhibitable mitochondrial pathway. J Biol Chem. 2001;276(15):12060-12067.

35. Shimizu S, Narita M, Tsujimoto Y. Bcl-2 family proteins regulate the release of apoptogenic cytochrome c by the mitochondrial channel VDAC. Nature. 1999;399(6735):483-487.

36. Cullen SP, Brunet M, Martin SJ. Granzymes in cancer and immunity. Cell Death Differ. 2010;17(4):616-623. 
37. Migueles SA, et al. Lytic granule loading of CD8+ T cells is required for HIV-infected cell elimination associated with immune control. Immunity. 2008;29(6):1009-1021.

38. Choy JC. Granzymes and perforin in solid organ transplant rejection. Cell Death Differ. 2010;17(4):567-576.

39. Jacquemin G, et al. Granzyme B-induced mitochondrial ROS are required for apoptosis. Cell Death Differ. 2015;22(5):862-874

40. Martinvalet D, Zhu P, Lieberman J. Granzyme A induces caspase-independent mitochondrial damage, a required first step for apoptosis. Immunity. 2005;22(3):355-370.

41. Bladergroen BA, et al. Expression of the granzyme B inhibitor, protease inhibitor 9, by tumor cells in patients with non-Hodgkin and Hodgkin lymphoma: a novel protective mechanism for tumor cells to circumvent the immune system? Blood. 2002;99(1):232-237.

42. Medema JP, et al. Blockade of the granzyme B/perforin pathway through overexpression of the serine protease inhibitor PI-9/ SPI-6 constitutes a mechanism for immune escape by tumors. Proc Natl Acad Sci USA. 2001;98(20):11515-11520.

43. Ansari AW, Temblay JN, Alyahya SH, Ashton-Rickardt PG. Serine protease inhibitor 6 protects iNKT cells from self-inflicted damage. J Immunol. 2010;185(2):877-883.

44. Belkaid Y. Regulatory T cells and infection: a dangerous necessity. Nat Rev Immunol. 2007;7(11):875-888.

45. Hoffmann P, Ermann J, Edinger M, Fathman CG, Strober S. Donor-type CD4(+)CD25(+) regulatory T cells suppress lethal acute graft-versus-host disease after allogeneic bone marrow transplantation. J Exp Med. 2002;196(3):389-399.

46. Brusko T, Bluestone J. Clinical application of regulatory T cells for treatment of type 1 diabetes and transplantation. Eur $J$ Immunol. 2008;38(4):931-934.

47. Bradley JA. Transplant tolerance by Treg therapy. Am J Transplant. 2014;14(1):5-6.

48. Tang Q, Lee K. Regulatory T-cell therapy for transplantation: how many cells do we need? Curr Opin Organ Transplant. 2012;17(4):349-354.

49. Efimova OV, Kelley TW. Induction of granzyme B expression in T-cell receptor/CD28-stimulated human regulatory T cells is suppressed by inhibitors of the PI3K-mTOR pathway. BMC Immunol. 2009;10:59.

50. Putnam AL, et al. Expansion of human regulatory T-cells from patients with type 1 diabetes. Diabetes. 2009;58(3):652-662.

51. Wang Y, et al. Regulatory $\mathrm{T}$ cells require mammalian target of rapamycin signaling to maintain both homeostasis and alloantigen-driven proliferation in lymphocyte-replete mice. J Immunol. 2011;186(5):2809-2818.

52. Li B, et al. Noninvasive diagnosis of renal-allograft rejection by measurement of messenger RNA for perforin and granzyme B in urine. NEngl J Med. 2001;344(13):947-954.

53. Muthukumar T, et al. Messenger RNA for FOXP3 in the urine of renal-allograft recipients. N Engl J Med. 2005;353(22):2342-2351.

54. Read S, et al. Blockade of CTLA-4 on CD4+CD25+ regulatory T cells abrogates their function in vivo. J Immunol. 2006;177(7):4376-4383.

55. Gautron AS, Dominguez-Villar M, de Marcken M, Hafler DA. Enhanced suppressor function of TIM-3+ FoxP3+ regulatory T cells. Eur J Immunol. 2014;44(9):2703-2711.

56. Huang CT, et al. Role of LAG-3 in regulatory T cells. Immunity. 2004;21(4):503-513.

57. Kornete M, Sgouroudis E, Piccirillo CA. ICOS-dependent homeostasis and function of Foxp3+ regulatory T cells in islets of nonobese diabetic mice. J Immunol. 2012;188(3):1064-1074.

58. Turner JE, et al. CCR6 recruits regulatory T cells and Th17 cells to the kidney in glomerulonephritis. J Am Soc Nephrol. 2010;21(6):974-985.

59. Paust HJ, et al. CXCR3+ Regulatory T Cells Control TH1 Responses in Crescentic GN. J Am Soc Nephrol. 2016;27(7):1933-1942.

60. Förster R, Davalos-Misslitz AC, Rot A. CCR7 and its ligands: balancing immunity and tolerance. Nat Rev Immunol. 2008;8(5):362-371.

61. Chung BH, Kim KW, Kim BM, Doh KC, Cho ML, Yang CW. Increase of Th17 Cell Phenotype in Kidney Transplant Recipients with Chronic Allograft Dysfunction. PLoS One. 2015;10(12):e0145258.

62. Grimbert P, et al. T-cell phenotype in protocol renal biopsy from transplant recipients treated with belatacept-mediated co-stimulatory blockade. Nephrol Dial Transplant. 2011;26(3):1087-1093.

63. Liston A, Gray DH. Homeostatic control of regulatory T cell diversity. Nat Rev Immunol. 2014;14(3):154-165. 